\title{
The NRL Geospatial Hub for Ocean Sensor Processing and Collaboration
}

\author{
Bruce Lin, Elias Ioup, John Sample, Ph.D. \\ Naval Research Laboratory \\ Stennis Space Center, MS 39529
}

\begin{abstract}
The Naval Research Lab Geospatial Hub, or GHub, provides a rapidly deployable, scalable platform for geospatial product collaboration. The GHub is not only a content management system with a web-based interface, but also hosts a variety of content-driven geo-services for tightly integrated, but loosely coupled tool interaction. The GHub presents a rich SOAP API to allow third party tools to build custom integration based on need. The contentdriven presentation of data in GHub makes it an ideal element for common information space architectures. The design of the GHub data store is especially suited to heterogeneous data types, such as those found in sensing of the ocean environment. By building tool-level integration with GHub, an architecture inherits peer-to-peer data sharing, disconnection robust synchronization between GHub nodes, a high-performance tile-based map server, and built-in product versioning.
\end{abstract}

Index Terms-Distributed information systems, Geographic information systems GIS, common information space, web services, mapping, ocean characteristics

\section{OCEAN CHARACTERIZATION}

Characterization of the ocean environment involves the ingestion and fusion of multiple sensor outputs, such as side-scan acoustic imagery and ocean profile measurements. These sensor outputs are typically multidimensional in structure with geospatial, temporal, and sometimes multi-spectral components. During surveybased missions or tactical environmental characterization, this sensor data is often shared between several analysts as they gather and process data on-scene [BML07].

A first step in managing data in this highly collaborative environment is determining a logical data model. At this stage of modeling we are more concerned with the interrelation of data types than the data types themselves. Taxonomy classes can be defined for this realm of sensor-ingested ocean characteristics. An evolving DoD unified message exchange standards effort, Universal
Core (UCORE). One part of UCORE defines a toplevel taxonomy for entities and events that lie within the interest of the DoD enterprise [oD10]. UCORE defines a set of events as shown in Figure 1.

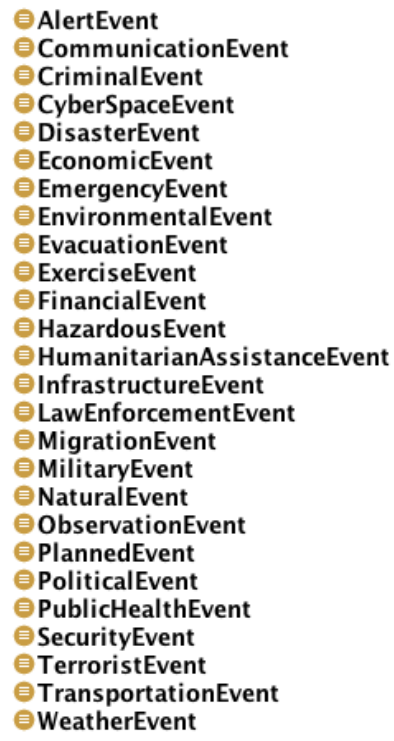

Fig. 1. Top-level UCORE 2.0 taxonomy for events.

A natural way to incorporate sensor observation concepts is to subclass ObservationEvent with a SensorobservationEvent. A SensorobservationEvent has a geospatial context, a temporal context, and associated ObservationData. The conceptual model can be described by a joining of data type and the data itself. The data type for a digital observation can be used to describe input and output compatibility between processing actions. This data type model defines three elements.

1) Parameter: The real world quantity or quantities 
being observed. For ocean characteristics, this includes parameters such as surface water temperature, salinity, water depth, etc.

2) Representation: The geometric representation of the sensed data. Sensors may record measurements in representations such as samples along Z-axis (profiles), a digital image, a field of points, a time series, or a collection of polygonal coverages.

3) Digital Format: The encoding of the sensed data in a digital format. Typical digital sensor formats include HDF, NetCDF, CSV, etc.

As a set of ontology classes and relations, an instance of a sensor data type is a triplet collection of individuals from each element class. The example in Figure 2 depicts an instance of a sensor data type as a triplet of bathymetry (parameter), grid (representation), and CHRTR (digital format).

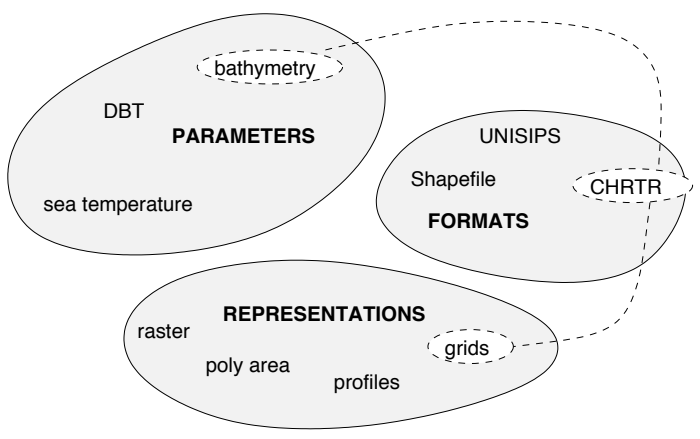

Fig. 2. The sensor data type conceptual model

This concept of sensor data type allows a suite of client tools to determine type compatibility beyond what file format, often simply data file extension, can communicate. For instance, within a NetCDF-formatted data file, with a .nc extension, can reside many parameters simultaneously. This is a common case a sensor platform will record with many sensors at the same time. Similarly, oceanographic models will produce many output variables within the same NetCDF file. While the storage format is observed from the file extension, it is useful to externally describe the parameters and geometric and temporal representations.

Not only can the type of a sensor product be captured, but also the input and output interfaces of a processing tool or algorithm. Take for example a program that is able to read a GeoTIFF-formatted image, and apply a image detection and classification algorithm to find man-made objects on the ocean floor, versus natural objects (e.g., rocks). The algorithm within the program is designed to operate on side-scan acoustic imagery, not optical. A GeoTIFF image has no intrinsic means to describe the image contents or the means by which the observations are made. The operator of the tool would need prior knowledge to only pass GeoTIFF imagery of the right sensor source to the algorithm. The data type model applied to both the GeoTIFF data file allows a mediating system to preempt processing if the data types are incompatible.

This simple factoring of the type of a data product empowers a oceanographic data processing system to add in process automation at many points, freeing the human operator from more mundane tasks of file organization and applying naming conventions to infer contents. The NRL Geospatial Hub is one example of mediating software, through the use of geospatial, data, and semantic services can be used to facilitate the sharing of ocean data.

\section{Features in the Geospatial Hub}

The GHub's facilities to support versioning, enterprise-level sharing, and data discovery make it an ideal solution for supporting many different kinds of ocean environment characterization. Data within the GHub is formed into logical groupings called datasets. Internally, however, the data is stored wholly within the file system, which is obscured to the end user. Each dataset is placed within a folder at the root level of the GHub. A folder can contain additional folders or any number of datasets, with each dataset representing one or more files. Each dataset is used to generate an individual page displaying all of the information stored within GHub pertaining to the dataset in question, as well as providing for RSS feeds alerting the user to changes within the dataset. A datasets page also contains download links, comments, and any additional functionality extended to the dataset type.

Because GHub is file-format agnostic, it can be used to store and retrieve any type of data. Any type of data can be organized into a dataset and stored within GHub. In addition to the basic functionality GHub extends to all datasets, certain datasets provides additional services for specific dataset types.

Besides simple storage and retrieval, the functionality GHub extends to all data entered within it consists of: 
1) version management,

2) basic metadata,

3) keyword search, and

4) synchronization

For versioning purposes, a unique timestamp is added to all data entered within the GHub to prevent any data conflicts. These versions are retained even after more current versions of the data are uploaded into GHub; effectively it is impossible to permanently delete any changes within the GHub.

The metadata associated with each file includes information such as: the title of the dataset, the creator, the classification, a description, a list of topics and associated keywords, and a bounding box depicting the geospatial area of interest for the dataset in question.

The keyword search is robust, allowing the user to specify which phrases must appear within their results, or if a different phrase is to be excluded. Further, the user can search using a set of latitude/longitude points in order to return all of the data pertaining to an area of interest, or even select an area upon an interactive map for searching.

Finally, GHub allows for more compact, remote versions of the main GHub to operate using a subset of the datasets within the main GHub, with alterations made in either GHub synchronized together. This synchronization is both automatic and built to operate over limited bandwidth networks with resumable transfers in case of interruptions.

As an example of the additional services provided to certain datasets, GHub indexes the internals of Office documents (Word, Excel, PDF, etc.) and expose them to search. Images (JPEG, GIF, PNG) provides preview functionality on their individual dataset page. Shapefiles have geospatial web services support in the form of WMS, WFS, and KML and links to utilize each of these features likewise are available within the dataset page.

The RDF dataset within GHub provides an index over generic, geospatial, and temporal keywords within a file and expose them to search. Further a SPARQL endpoint is created to provide SPARQL access into the stored RDF documents.

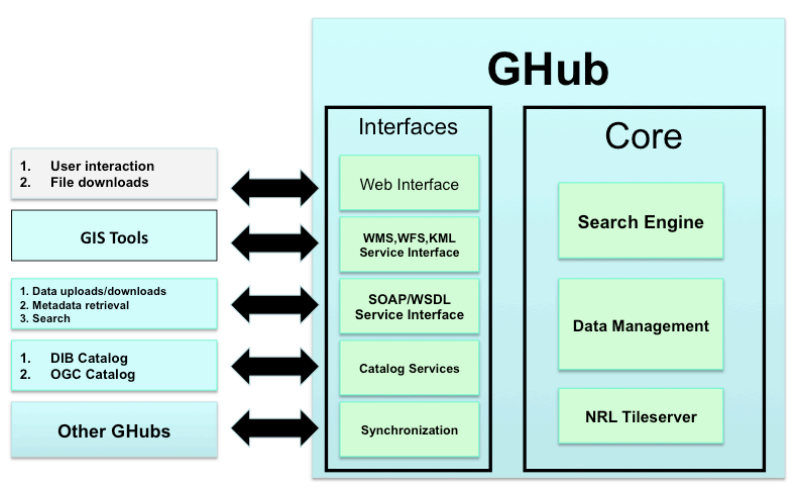

Fig. 3. Geospatial Hub system architecture

\section{INTEGRATION TECHNIQUES}

An ocean data processing client, either GIS software or process/analysis tools, have multiple options to exploit the services available in a GHub instance. It is not necessary to completely implement all of the services. A GHub client may take an "a la carte" approach selecting an integration path.

\section{A. Map Services}

A basic feature of GIS software and programs with GIS-like capability is a layered-map. On the Internet, it is nearly trivial to employ maps and map-widgets in an application. However, in a limited connectivity environment, such as on a oceanographic survey, access to high-resolution maps and map products is not possible, or bandwidth prohibitive. Moreover, commercial map products do not provide a useful context over water areas. The Geospatial Hub's tile service provide all standard National Geospatial Agency (NGA) map products in a uniform projection and tiling scheme.

Client software may elect to consume tile-based maps from the GHub's map server in a raw tile protocol, web-map service (WMS), or Keyhole-markup (KML). Many applications have the built-in ability to consume map services in the latter two protocols already, such as Google Earth ${ }^{\mathrm{TM}}$, NASA World Wind, and ESRI ArcGIS $^{\mathrm{TM}}$.

\section{B. Data Management}

A processing environment for oceanographic data may generate a file, or collection of files that can be added 


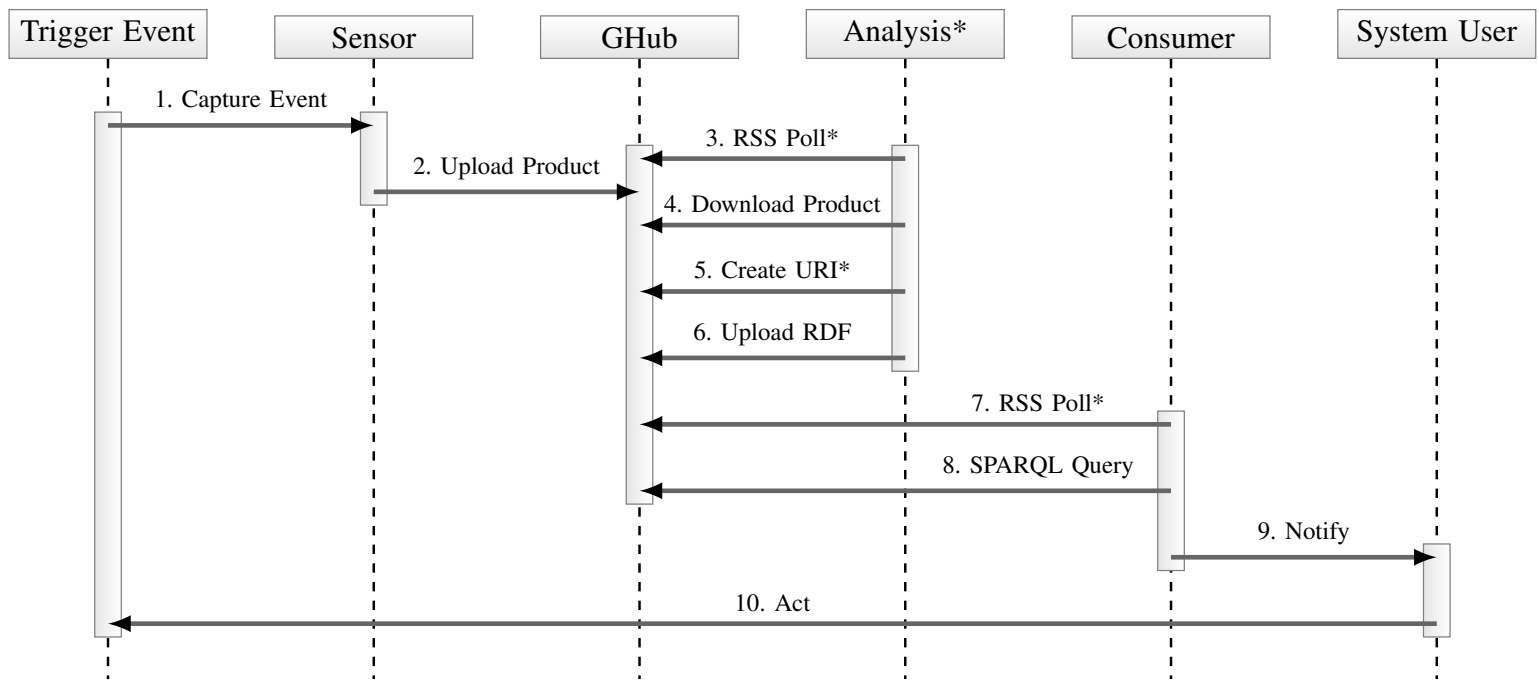

Fig. 4. Sequence diagram, trigger event loop

to the GHub data repository. Although this is possible through the web-based interface, the preferred integration mechanism is using the SOAP interface functions to programmatically send data into GHub. Such tight integration provides a much more seamless user experience. However, this method requires integration programming, and a practically, a development platform that is SOAPfriendly. Java ${ }^{\mathrm{TM}}, \mathrm{C}++$, and Microsoft .Net $^{\mathrm{TM}}$ are popular environments. As intermediate solutions, GHub "helper" tools can be incorporated into the data publication process.

Once added to GHub, a oceanographic data set are automatically indexed. A supported geospatial format is read and the bounds are computed or extracted and made searchable. Keywords can accompany the data set as tags for discoverability. The GHub search is also access through web-based or SOAP interfaces. Data sets in the GHub can be arbitrarily organized in a folder structure to match the organizational needs of the user.

\section{Geospatial Data Services}

An enhanced feature of some geospatial data types in GHub is the ability to automatically create service endpoints. The following data formats are supported:

1) $\mathrm{KML}(. \mathrm{kml})$ : KML features are simply served again as a KML service

2) NetCDF (.nc): Coverage-based NetCDF files of certain ocean parameters can be previewed from a web mapping service (WMS). Also, sub-coverages may be extracted via a web coverage service (WCS)

3) ESRI $^{\mathrm{TM}}$ Shapefile (.shp): An WMS preview service is created to render the points, lines, and polygons residing in the file. Also, the geometry within the Shapefile is converted to a web feature service (WFS) to deliver geography markup (GML) and as a KML feature service.

\section{Semantic Web Integration}

Ontologies capture the structure and relationships between concepts in a knowledge domain. When an ontology is expressed in the web ontology language (OWL), a reasoning engine can be applied to infer new associations. Semantic statements made RDF/OWL documents have several applications to ocean data management.

1) Data type constructions described in Section I can be expressed as semantic statements. As a result, a semantic service can be used to draw inferences to complete partial data type expressions. For instance, if part of a data type is known, a semantic query could be used to complete the remainder. This can be useful in automatic algorithm selection.

2) Ontologies can be used to flexibly express the associations between sensor data types with metadata 
items. Instead of coding this logic into each client, this can be captured and shared at an enterprise level.

3) Processing rules can be expressed in an ontology. As an alternative to stove piped processing chains, an ontology of processing steps could assist in service orchestration [LWP ${ }^{+}$], [WPJ07].

In a more established environment with reliable connectivity, an instance of GHub can support much more data and client connectivity. The GHub web interface allows users to freely browse the data repository with only a web browser, as well as preview some kinds of geospatial data by the GHub with a browser-based map client. Figure 4 gives a more intricate event sequence view of the ocean product fusion process.

\section{DISCUSSION}

GHub can support different deployment strategies. For instance, GHub can be deployed on an ad hoc network of laptops. Here, several analysts with servicesenabled software tools can employ GHub transparently. Products that are published to the GHub data store can be automatically shared with other clients or automatically fed into other processes.

As depicted in Figure 5, the process flow for Level 1 fusion process is described simply.

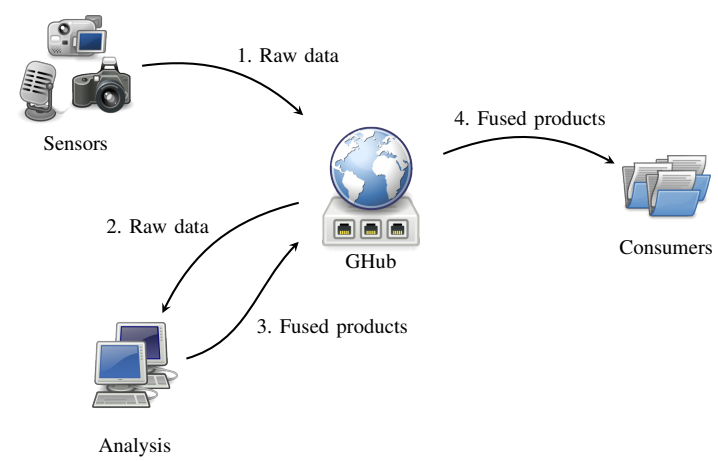

Fig. 5. GHub-centric view of ocean sensor processing flow

1) A heterogeneous collection of sensor hardware gathers raw data and stores them directly in the GHub.

2) Using GHub's publication interface, one ore more analyst nodes can begin work on performing data conditioning and/or data fusion.
3) All products and metadata are fed back into the GHub.

4) With the same publication interface, product consumers are notified of updates which can then be retrieved for on-scene knowledge updates, decision making, or archival.

In this paper, we described how the GHub services can be integrated with a several thick client tools such as Google Earth, NASA World Wind, Arc Desktop, and other ocean environmental tools employed by the US Navy.

\section{REFERENCES}

[BML07] A. Berni, D. Merani, and M. Leonard. Network-enabled information fusion in support of underwater research. 2007.

$\left[\mathrm{LWP}^{+}\right]$R. Ladner, E. Warner, F. Petry, K.M. Gupta, P. Moore, D.W. Aha, and K. Shaw. Case-based classification alternatives to ontologies for automated web service discovery and integration. In Proceedings SPIE Defense \& Security Symposium, volume 6201, pages 620117-1.

[oD10] Department of Defense. Universal Core 2.0. www.ucore.gov, 2010.

[WPJ07] A. Wun, M. Petrovic, and H.A. Jacobsen. A System for Semantic Data Fusion in Sensor Networks. 2007. 